The non-cough-up tubes (Fig. $3 E$ and $F$ ) have a low bulbous extremity which holds in the trachea. I have been using these tubes for the past four years, and so far in over three hundred cough-up cases not a single one has been coughed out after introduction. These tubes have put a stop to much of the anxiety which accompanies intubation.

The post tube (Fig. $3 G$ ) has a device which can be screwed into the tube through the tracheal fistula. The tube is first introduced and scratched through the wound so that the scratch mark corresponds to ihe tracheal fistula, then it is removed and a thread hole bored to accommodate the post.

The bridge tube (Fig. $3 H$ and $I$ ) I have employed after laryngofissure. The tube is introduced through the mouth, and when in position the bridge is attached to it. Strips of adhesive plaster cross this bridge and are strapped tightly to the sides of the neck to make posterior pressure, so that the anterior fissure would heal primarily. I have discarded their use as I have resorted to dilatation only during the past five years.

Low tracheal and bronchial tubes are used only in low tracheal and bronchial diphtheria. I have been using them for the past year and so far have had gratifying results.

The tunnelled sound with obturator is employed in tracheal cannula cases when it is necessary to dilate the larynx above the cannula. The sound is tunnelled so that the patient can get air during the process of dilatation. These are made from urethral sounds. After dilatation has been accomplished this tunnelled sound is passed, the tube attached to the obturator and intubation performed. All of these tubes and apparatus are made by George Ermold of New York,

24 West Fifty-Ninth Street.

\section{NEW INSTRUMENT FOR THE REMOVAL OF ADENOIDS}

Marvin McTyeire Cullom, A.B., M.D., Nashville, Tenn. Fellow of the American College of Surgeons

The best instrument for the removal of adenoids is the curet. It has two drawbacks: the liability of losing the growth, and the possibility of stripping down the mucous

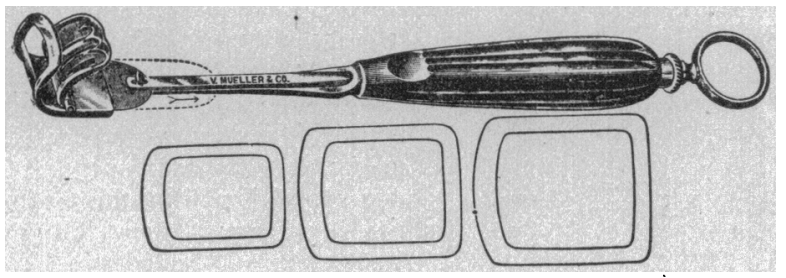

Fig. 1.-Instrument for the removal of adenoids.

membrane on the back wall of the pharynx. Aside from these defects, no instrument has yet been devised which is superior to it.

I have attempted to devise an instrument which will preserve the good qualities of the curet and at the same time overcome these two difficulties.

The instrument presented has all the features of the curet. In addition it has a flexible blade which cuts the growth at the back, and a movable cage over the fenestrum of the curet which permits the adenoid to project through the instrument and enables it to get down to the very base of the growth. The flexible blade is worked by an arm running through the handle to a thumb ring. In removing the growth the instrument is thrust forward until it touches the vomer; it is then pushed down until it settles snugly around the growth, the flexible knife is thrust home with the thumb, and the adenoid is removed with a free sweep just as with a curet.

The flexible knife does not cut entirely through the growth, but the final severance is made with the sharp edge of the curet.
The growth usually comes away en masse. The finger is then introduced to make sure that the removal is complete. It can be used as an ordinary curet by drawing back the blade.

In the first few operations in which I used the instrument, I was rather startled by the size of the growth removed. It

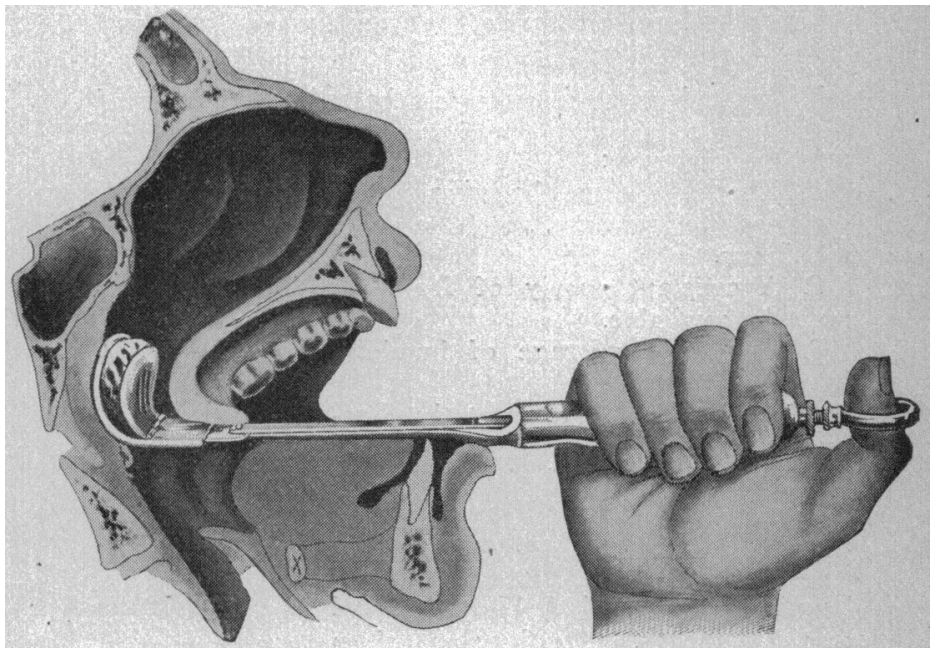

Fig. 2.-Method of use

was because I was not accustomed to seeing the entire adenoid mass in one piece.

214 Hitchcock Building.

\section{AN IMPROVED PESSARY AND TAMPON HOLDER}

\section{Worth Norton, M.D., Los Angeles}

As will be seen from the illustration, which is about threeeighths actiual size, this pessary is simple in construction and practical in application. Figure 1 shows the instrument ready for insertion, while Figures 2 and 3 picture the pessary and introducer separately. Its usefulness as a support is due to the fact that it will remain in place and hold the cervix in its normal position. It is worn without discomfort as its shape conforms to that of the vagina. The pessary is constructed of aluminum and is light, indestructible, and can be sterilized by boiling. The fenestrations laterally permit the entrance of the soft parts, doubly securing the instrument in position. As any support is needed only when the patient is on her leet, the pessary can easily be removed at bedtime and reinserted in the morning. A slightly longer and wider pessary is required for a badly lacerated case, while an atrophic, shortened vagina is best

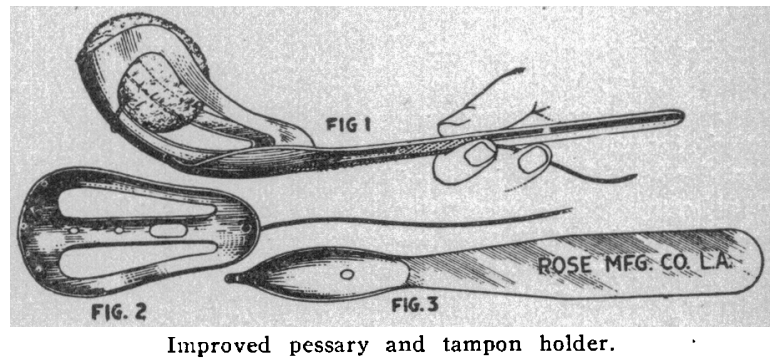

served by a shorter but slightly wider pessary. After being instructed by the physician, the patient can readily remove and reinsert the pessary.

This instrument has given good results in retroversion, prolapse, cystocele and general pelvic relaxation.

Medicated cotton secured within the pessary serves as a method of prolonged application to the cervix and adjacent tissues.

507 Bumiller Building. 